\title{
Remote Microsurgery System for Deep and Narrow Space - Development of New Surgical Procedure and Micro-robotic Tool
}

\author{
Koji Ikuta, Keiji Sasaki, Keiichi Yamamoto, and Takayuki Shimada \\ Department of Micro System Engineering, Graduate School of Engineering, \\ Nagoya University, Furo-cho, Chikusa-ku, Nagoya, Aichi, 464-8603, Japan \\ Tel: +81 52-789-5024, Fax: +81 52-789-5027 \\ ikuta@mech.nagoya-u.ac.jp
}

\begin{abstract}
We developed a new medical operation procedure and robotic tool of microsurgery in deep and narrow site. It enables us to operate a difficult microsurgery that conventional method can't be achieved. Our system consists of flexible slave micro manipulators which can enter deep site of human body like a flexible catheter and master manipulators. Owing to difference of their sizes, it enables to convert micro motion of slave into natural size motion of master. Finally, both feasibility and effectiveness of the total system were verified experimentally.
\end{abstract}

\section{Introduction}

The target of this study is microsurgery in deep, narrow sites of the body, which are currently the most difficult areas to perform minimally invasive surgery. Typical examples are neurosurgery, head and neck surgery in otolaryngology, and microsurgery on esophageal cancer in the outer wall of the esophagus, an area that is not accessible with an endoscope through the mouth. We proposed and developed both a new method of microsurgery and surgical tools. Through a two-step development, a system was successfully created in which a slave manipulator with seven degrees of freedom in movement is remotely controlled from outside the body. The driving system is the size of a notebook computer and weighs approximately $1 \mathrm{~kg}$. It can be hung on a bedside stand when in use. Dramatic reductions in size and weight from the current surgical robot prototype were accomplished. Finally, the effectiveness of the system was verified in animal experimentation.

\section{Sophisticated Medical Treatment and Cases in Which Microsurgery Is Impossible}

The current method of microsurgery has the following problems:

1. It is not applicable to deep sites of the body or the nasal cavity, which are difficult to access

2. Microscopic manipulation cannot be done at a level beyond the skill of the surgeon.

3. It requires a large incision. 
In current medical treatment, there are other types of surgery that are very difficult or nearly impossible to perform, such as those on the esophagus and other digestive organs, those on the spleen and other organs located deep inside the body, and those on fetal organs. This is because there are critical organs, such as the lungs and the heart, that are in the approach path to the site to be operated.

Factors that prevent surgery are shown below:

1. Critical organs, such as the lungs and the heart, that are in the approach path must not be touched

2. Even when the site is accessible, a severely restricted space will not allow for any surgery in which forceps are inserted from the outside.

3. The surgery itself is very difficult because it is extremely microscopic.

There are also surgeries that are performed under X-ray exposure. In these cases, remote medical treatment will be necessary to protect surgeons from exposure to $\mathrm{X}$ rays[1]. Among remote microscopic surgical systems, there are the Tele Microsurgery System by Mitsuishi et al.[2] and the HUMAN Manipulator System by Kan et al. [3].

Most of these systems are basically used in open surgery to perform microscopic manipulation remotely. In other words, they are the current remotely controlled surgical systems. Therefore, it is very difficult to apply them to the cases mentioned above.

\section{Proposal of a New Method of Microsurgery}

The new method of microsurgery we propose is shown in Fig. 1.

1. As in a catheter procedure, a guide tube is inserted following after insertion of a guide wire and is threaded through the space between organs to the site to be operated.

2. The guide wire is withdrawn, and the slave micromanipulator is inserted along the tube to the site to be operated. More slave manipulators and an endoscope may be inserted in the same way as necessary.

3. While monitoring the images from the endoscope, the master manipulator is used in the microsurgery performed by the slave manipulator in the restricted space.

To use the new method of microsurgery in deep sites of the body and in restricted space, we propose the remote microsurgery system as shown in Fig. 2. In this system, the microscopic procedure is performed by a slave manipulator controlled through a master manipulator, which is several times larger in scale than the slave manipulator, by the operator. Furthermore, the slave manipulator allows a winding approach to be taken, going around organs to reach the site to be operated in the patient's body.

The features of the system are shown below:

1. Flexible movement of the slave manipulator makes it possible for microsurgery to be performed at deep sites of the body.

2. Minimally invasive surgery can be performed since no incision is required.

3. The difference in size between the master and slave manipulators makes it possible to perform microsurgery at a level beyond the skill of the doctor. 

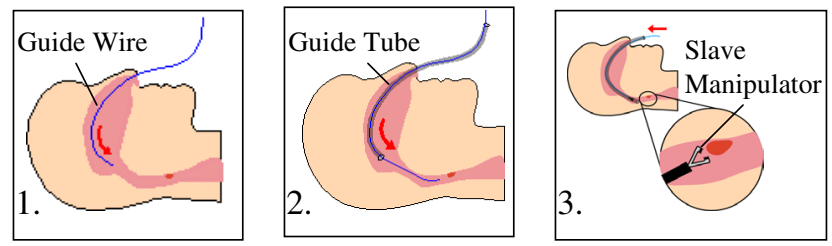

Fig. 1. New Method of Microsurgery in Deep and Narrow Site

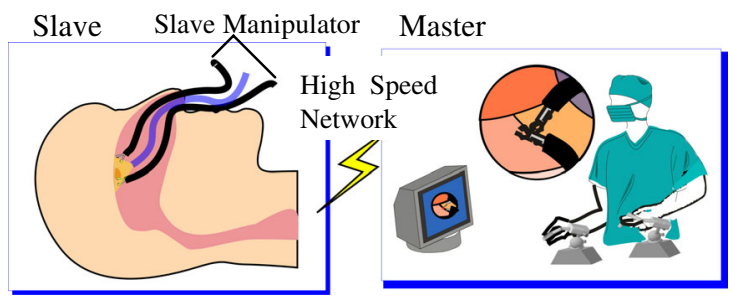

Fig. 2. Proposed Concept of Remote Microsurgery System

\section{Preparation of the System}

\subsection{Requirements for the Slave Mechanism}

Requirements for the slave mechanism are shown below:

1. Miniaturized tip manipulator

2. Able to follow a winding approach to the site

To meet requirement 1 , the degree of freedom in movement of the tip manipulator was limited to five (the smallest number considered necessary to be useful as a microsurgical tool): (1) translation, (2) torsion, (3) grasping, (4) base joint, and (5) tip joint. At the same time, the tip manipulator unit is to be separated from the driving member unit. As for translation and torsion, the slave manipulator was devised so that it can move its main body itself in such a way that translation and torsion is obtained at the tip manipulator.

To meet the requirement 2, a microwire transmission mechanism was adopted as a transmission mechanism from the driving member to the tip manipulator so as to make the intermediate portion of the slave manipulator flexible.

In general, the wire system can cause the following problems, which have been solved by adopting a noninterference microjoint mechanism and the S-Drive, which will be explained later, to compensate for any slack:

1. Redundant movement of multiple degrees of freedom is difficult

2. Slack in the wire

\subsection{Decoupled Microjoint Mechanism [4]}

Fig.3 is a diagram of the "decoupled microjoint mechanism". Since the wire for driving the tip joint always passes through the axis of the base joint, the path length of the 
driving wire remains constant regardless of the angle at which the base joint is bent. Therefore, there will be no interference of the base joint by the tip joint.

\subsection{S-Drive for Elongation Compensation}

Fig. 4 is a diagram of the S-Drive. The S-Drive compensates for any elongation in the wire to keep the wire's tension constant using a spring, which is based on TendonDrive Mechanisms by S.Hirose et al.[5] The drive also features the ability to apply the maximum amount of static friction between the wire and the pulley on the motor
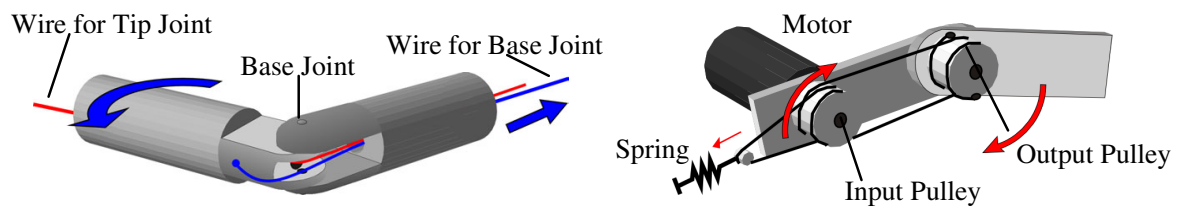

Fig. 3. Decoupled Microjoint Mechanism with 2D.O.F

Fig. 4. S-Drive Mechanism to compensate elongation after long term driving

Slave

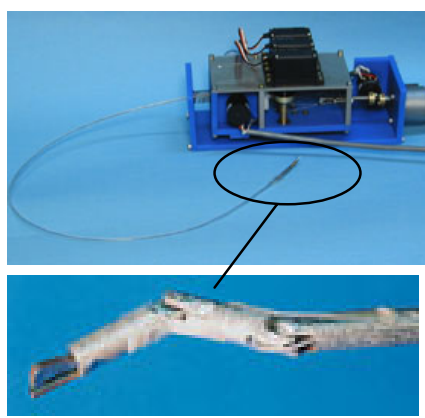

\section{Master}

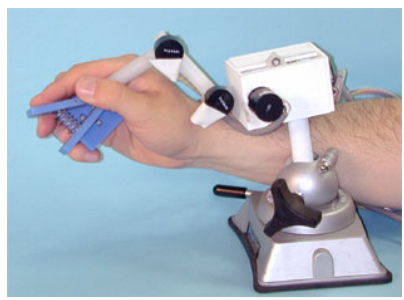

Fig. 5. First Prototype of Remote Microsurgery System (2000)

Slave

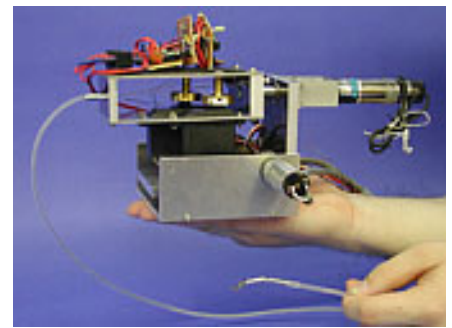

Master

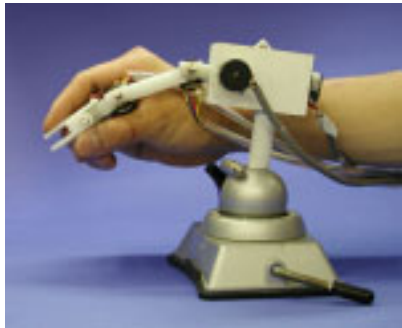

Fig. 6. Second Prototype of Remote Microsurgery System (2001) 
side using the spring. Therefore, adjustments to the spring will determine the maximum torque to be transmitted to the tip, thus functioning as a torque limiter.

\section{Development of Prototypes}

In this study, the first prototype used in the verification of the basic principle was created in 2000.[6] In the model (Fig.5), the tip manipulator of $3 \mathrm{~mm}$ has five degrees of freedom in movement (a grasping mechanism, two joints, translation, and torsion), each of which was successfully driven independently. The first prototype, however, could not be operated steadily over long periods of time due to an insufficient driving capability resulting from a lack of rigidity.

To improve stability so that the manipulator could be used in long-term animal experimentation, a second prototype for animal experimentation was created in 2001 (Fig. 6).[7]

With the second prototype, animal experimentation on a chicken was conducted, and the following results were obtained:

1. In the proposed method, deep sites of the body were accessed.

2. The manipulator was inserted from the neck of the chicken to the abdominal cavity, where the manipulator's capability for surgical manipulation was verified.

Based on the results, it was verified that access to deep and narrow sites of the body was possible and that microscopic manipulation was also possible at such sites. Thus, the effectiveness of the prototype system was verified. However, before going onto the clinical application in the next step, the following had to be realized:

1. Improvement in the driving properties and power

2. Increase in the degree of freedom in movement of the slave manipulator tip

3. Improvement in the operability of the master manipulator
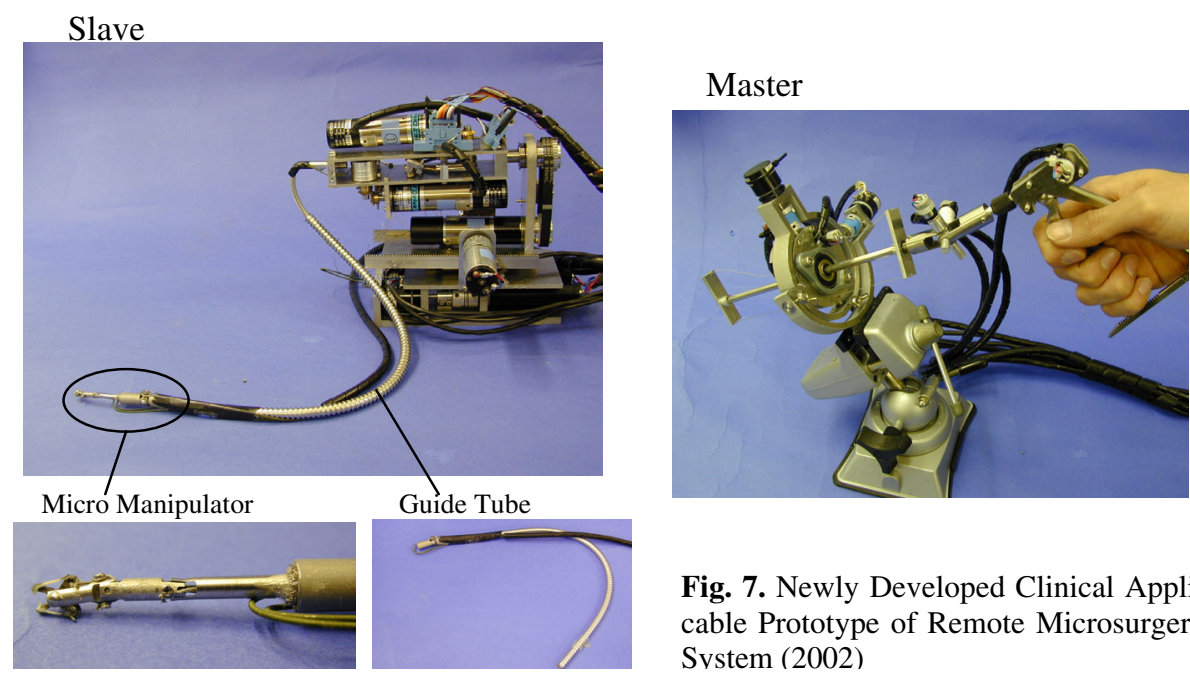

Fig. 7. Newly Developed Clinical Applicable Prototype of Remote Microsurgery System (2002) 


\section{The Third Prototype for Clinical Application}

Fig. 7 shows an external view of the prototype created for clinical application in this study. Pitch and yaw are added to this prototype to increase the degree of freedom in movement of the tip. Thus, the tip of the guide tube is provided with a total of seven degrees of freedom in movement.

The prototype features the following:

1. Increase driving capability through an improvement in wire transmission properties

2. Increase in the degree of freedom in movement through the introduction of a tip driving guide tube (seven degrees of freedom in movement at the tip)

3. Increase operability through an improvement in the master manipulator

4. Reduction in size and weight of the entire system

Each of the items above is explained below:

\subsection{Driving Member of the Slave Manipulator}

The driving member of the slave manipulator is a unit into which the actuators of the tip manipulator are integrated. The changes made from the second prototype are shown below:

\section{A guide tube driving system is added}

An actuator is newly added to the guide tube, and the driving system for the guide tube is made to be interchangeable with the driving system for the tip manipulator so that it would be able to cope with changes in the tip manipulator.

2. The amount of translation and torsion is increased

The amount of translation and torsion is increased to 1.5 times the current amount so as to extend the work area.

3. The size and weight of the system is reduced

Due to an optimized motor arrangement and materials, the size and the weight of the entire system are greatly reduced despite an increase in the number of motors and the size of the work area.

The properties of the new slave mechanism are compared to those of the current one in Table 1.

\subsection{Tip Micromanipulator}

The tip manipulator is a microscopic manipulator that performs operations in deep and narrow sites of the body. It is $3 \mathrm{~mm}$ in diameter, providing five degrees of freedom in movement: grasping, tip joint, base joint, translation, and torsion. The external view of the manipulator is shown in Fig. 8.

An improvement in the manipulator design and the use of an outer casing to increase the wire transmission capability greatly improve the driving property of the tip manipulator, providing sufficient power required for microscopic suturing in the body. 
Table 1. Comparison of the Characteristic

\begin{tabular}{|c|c|c|}
\hline & Clinical applicable prototype (' 02) & Previous Prototype("01) \\
\hline D. O.F. & 7 & 5 \\
\hline & Harmonic Drive $(\mathrm{RH}-5 \mathrm{~A}, 1 / 80) * 4$ & \\
\hline motor & Donotor (maxon 4.51 1/84) *2 & OC servomotor (Futaba $\$ 9450)$ \\
\hline & DCmotor (COPAL LC20G 1/100) *1 & \\
\hline Width $(\mathrm{mm})$ & 155 & 261 \\
\hline Extend (m) & 70 & 107 \\
\hline Height $(\mathrm{mm})$ & 130 & 120 \\
\hline Weight (g) & 1064 & 1087 \\
\hline Thrust movement $(\mathrm{mm})$ & 40 & 25 \\
\hline movable angle (deg.) & \pm 150 & \pm 90 \\
\hline Max speed Thrust $(\mathrm{m} / \mathrm{s})$ & 62 & \\
\hline Twist (rpm) & 55 & \\
\hline
\end{tabular}

\subsection{Additional Degrees of Freedom on the Tip of Guide Tube}

In the method proposed in this study, "guide tube" is defined as a tube used to secure the path of the slave manipulator. So that a tip manipulator could work in the target position, pitch and yaw are newly added to the guide tube. It is constructed so that a universal joint with a controllable tip is joined at the tip of a stainless steel flexible tube (Fig. 9).

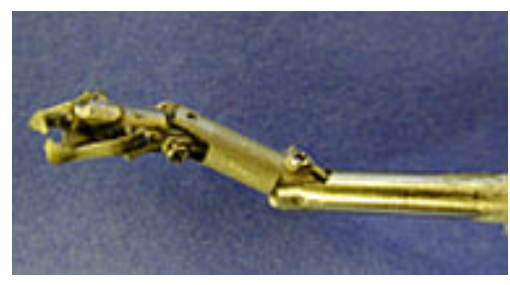

Fig. 8. Newly Developed Micromanipulator with 7D.O.F

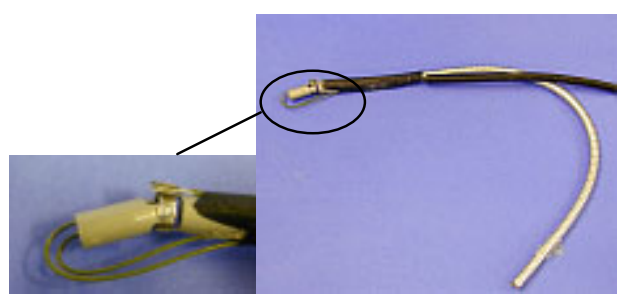

Fig. 9. Decoupled microjoint with 2D.O.F on the tip of guide tube

The system features the following:

1. Seven degrees of freedom in movement, including pitch and yaw, provided for the tip

The two new degrees of freedom in movement provided for the tip of the guide tube increase the number of degrees of freedom in movement at the tip portion to seven (together with the five degrees of freedom in movement of the manipulator). This greatly contribute to an increase in operability.

\section{Flexible body trunk}

The entire flexible construction of the body trunk is similar to that of a catheter, allowing the manipulator to be inserted and operated without damage to the human body. 


\section{A driving source placed outside of the body}

The wire mechanism allows the driving source to be placed outside of the body. This makes it an excellent system in terms of safety because there is nothing in the body that is electrically run.

\subsection{Master Mechanism Member}

The master mechanism is a control stick with which a doctor operates the system. Because microsurgery requires microscopic manipulation, which is very difficult to do, it takes a lot of time. Therefore, the master mechanism must be easy for the doctor to operate, causing little fatigue even in a prolonged procedure.

The master mechanism previously reported had the following problems:

1. Insufficient operability

2. Limited work area

As to item 1, because the previous master mechanism was of the same pen-shaped structure as the slave mechanism, holding and operating the master mechanism was very difficult. Thus, the problem of operability remained.

As to item 2, because the master and slave manipulators were of the same structure, their work areas were identical. This allowed for intuitive operation, but there was the same restriction with the degree of freedom in movement on the master side as on the slave side, forcing the doctor to operate it in an unnatural position. The restricted degrees of freedom in movement may have been the cause of stress in doctors.

To solve these problems, a forceps-shaped master is created for this study.

Features of the master are shown below:

\section{Increase in the degree of freedom in movement}

A tip driving guide tube is newly added to increase the number of degrees of freedom in movement to seven, and a corresponding change is made on the master side so as to control these seven degrees of freedom in movement.

2. Good operability, giving the doctor a sense of congruity

A structure shaped like forceps, which is a familiar object to doctors, allows the doctor to perform microsurgery with the same feeling as in laparoscopic surgery, which is becoming increasingly popular. Furthermore, fewer restrictions on the degree of freedom in movement eliminate operative restrictions, successfully providing good operability.

3. Good control of the slave

The degree of freedom in movement on the master side corresponds to that on the slave side, although the master has a different structure. This eliminates the need for coordinate transformation, thus creating a unique master that provides a feeling of intuitive operation, which is an advantage of masters of the same structure, as well as good operability, which is an advantage of masters of a different structure.

The corresponding degrees of freedom in movement on the slave side are shown in Fig. 10. The gimbals equipped with a locking mechanism senses the pitch and yaw of the guide tube and the forceps mechanism senses the grasping, two joints, translation, and torsion of the tip manipulator.

When operating the mechanism, the gimbals is used to control the pitch and yaw of the guide tube for the rough positioning of the tip. Then, the gimbals is locked and the 
rest of the forceps mechanism is used to drive the tip manipulator in five degrees of freedom in movement to perform microsurgery.
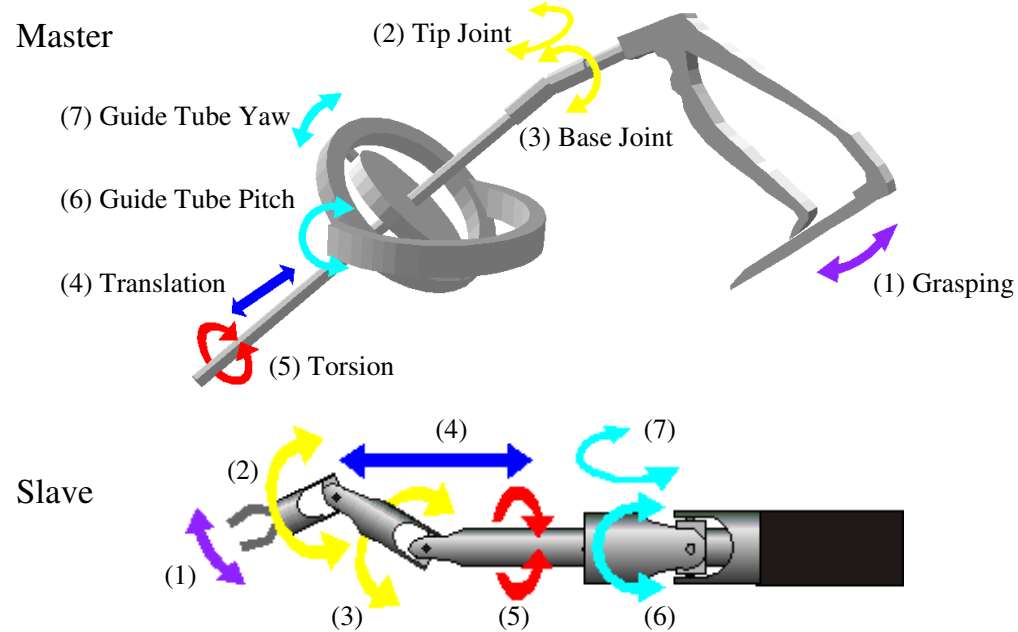

Fig. 10. Correspondence of D.O.F between Master and Slave Micromanipulator

\section{Verification Experiment}

To verify the functions of this system, experiments were conducted on animals. Livers of chicken were used to perform suturing, which is the most difficult of the basic procedures in microsurgery (Fig. 11). A curved, semicircular needle was used.
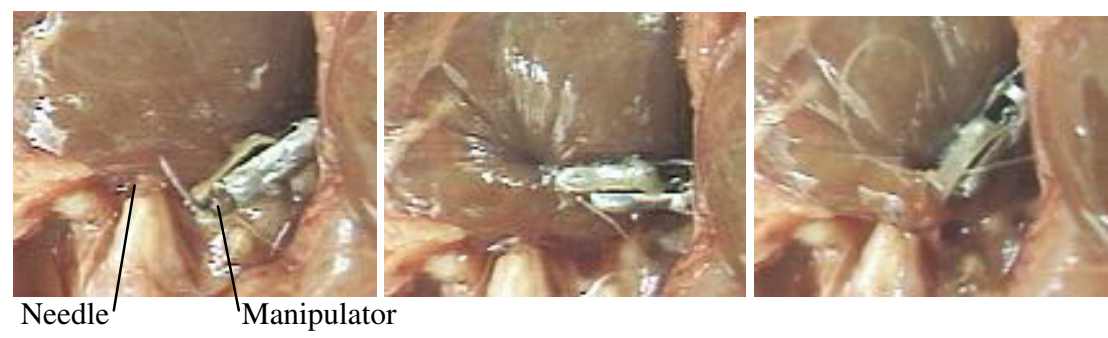

Fig. 11. Suture Experiment (from VCR image)

The results of the experiments showed that the series of operations performedtransferring a needle from the forceps, grasping the needle with the tip manipulator, moving it to a desired point, inserting the needle, and withdrawing the needle was successful. Thus, the effectiveness of the system was verified. 


\section{Conclusion}

We developed a new medical operation procedure and robotic tool of microsurgery in deep and narrow site. It enables us to operate a difficult microsurgery that conventional method can't be achieved. Our system consists of flexible slave micro manipulators which can enter deep site of human body like a flexible catheter and master manipulators. A prototype for clinical application was created. By improving the driving property, degree of freedom in movement, and operability of the tip manipulator, a prototype of a total system with a dramatically improved performance was created.

Especially in the tip manipulator, work efficiency and multipurpose applicability were successfully improved by increasing the degrees of freedom in movement of the tip to seven while keeping the size the same as that of the current tip $(3 \mathrm{~mm}$ in diameter). Furthermore, a verification experiment on a chicken verified that the basic procedures of microsurgery -ablation, partial excision, movement, fixing, and suturing- could be successfully performed.

\section{Acknowledgment}

The authors thank Dr. H.Iseki at Tokyo Woman's Medical University, Dr. D.Hashimoto and Dr. K.Shinohara at Saitama Medical School for their useful advice. We also acknowledge the assistance of all contributors.

\section{References}

1. K. Ikuta, T. Shimada, K. Ishizuka and Y. Nakayama, "Servo Forceps with Force Sensation for Remote Minimal Invasive Surgery", Proc. of 1999 JSME Conference on Robotics and Mechatronics (ROBOMEC 99), 1P2-10-004, 1999 (in Japanese).

2. M. Mitsuishi, et al "A Tele-Micro-Surgery System", Proc. of the 8th Annual Conference of the Japan Society of Computer Aided Surgery (JSCAS 99), pp.123-124, 1999 (in Japanese).

3. K. Kan, et al, "An Operation Experiment of HUMAN-Manipulator Prototype System for Neurosurgery", Proc. of the 8th Annual Conference of the Japan Society of Computer Aided Surgery (JSCAS 99), pp.105-106, 1999 (in Japanese).

4. K. Ikuta, F. Higashikawa and K. Shimoya, "Study on High Performance Hyper Endoscope", Proc. of 1998 JSME Conference on Robotics and Mechatronics (ROBOMEC 98), 1AIII2-5, 1998 (in Japanese).

5. Shigeo HIROSE, Yasuyuki UCHIDA, Richard CHUConsideration of New Tendon-driven Mechanisms, Proc. of 1998 JSME Conference on Robotics and Mechatronics (ROBOMEC 98), 1CI2-3, 1998 (in Japanese).

6. K. Ikuta, T. Shimada and K. Sasaki , "Study on Remote Microsurgery System", Proc. of 2000 JSME Conference on Robotics and Mechatronics (ROBOMEC 00), 2A1-13-017, 2000 (in Japanese).

7. K. Ikuta, K. Sasaki and T. Shimada, "Prototype and in vivo surgical experiment of Remote Microsurgery System", Proc. of 2001 JSME Conference on Robotics and Mechatronics (ROBOMEC 01), 2P1-D6, 2001 (in Japanese). 